\title{
Editorial
}

\section{Ultra-Wideband Antennas}

\author{
James Becker, ${ }^{1}$ Dejan Filipovic, ${ }^{2}$ Hans Schantz, ${ }^{3}$ and Seong-Youp Suh ${ }^{4}$ \\ ${ }^{1}$ Electrical \& Computer Engineering Department, College of Engineering, Montana State University, \\ 610 Cobleigh Hall Bozeman, MT 59717, USA \\ ${ }^{2}$ Department of Electrical and Computer Engineering, University of Colorado, Boulder Campus Box 425, \\ Boulder, CO 80309-0425, USA \\ ${ }^{3}$ The Q-Track Corporation, 515 Sparkman Drive NW, Huntsville, AL 35816, USA \\ ${ }^{4}$ Intel Corporation, 2200 Mission College Boulevard, Santa Clara, CA 95054-1549, USA \\ Correspondence should be addressed to Hans Schantz, h.schantz@q-track.com \\ Received 22 May 2008; Accepted 22 May 2008
}

Copyright (c) 2008 James Becker et al. This is an open access article distributed under the Creative Commons Attribution License, which permits unrestricted use, distribution, and reproduction in any medium, provided the original work is properly cited.

Over the last few years much has been published about the principles and applications of electromagnetic waves with large relative bandwidth, or nonsinusoidal waves for short. The next step is the development of the technology for the implementation of these applications. It is generally agreed that the antennas pose the most difficult technological problem.

\section{Henning F. Harmuth, Antennas and Waveguides for Nonsinusoidal Waves, New York: Academic Press, 1984, p. xi.}

More than twenty years after Harmuth's observations on the difficulties posed by UWB antenna design and six years after the FCC authorized ultra-wideband (UWB) systems, a variety of UWB products are nearing wide-scale commercialization. Antenna designers and engineers have solved the UWB antenna problem in many ways, yielding compact antennas well suited for a variety of applications. Unlike in previous decades when UWB antenna progress came in fits and spurts, today there is an active and growing community of UWB antenna designers sharing their insights and designs at professional conferences, trade shows, and on the pages of technical journals.

A keyword search of Google Patents for "UWB antenna" provides quantitative data to capture these qualitative trends. This metric is somewhat crude and may overlook certain broadband antenna designs not explicitly labeled as "UWB." Similarly, this search may include UWB systems or services that merely mention "UWB antennas." Still, this analysis provides an interesting look at trends and activity in the area of UWB antennas.
Broadband and UWB antennas date back to the earliest days of radio [1]. Harmuth and others pioneered the use of impulse or "nonsinusoidal waves" in the 1970's and 1980's [2]. The Time Domain Corporation and Multi-Spectral So lutions, Inc., were both formed in the late 1980's to commercialize UWB technology.

The US Defense Advanced Research Projects Agency (DARPA) sponsored a review of UWB technology in 1990 in the context of radar applications [3]. This investigation was controversial, upholding the merit and value of UWB technology in general, while dismissing some of the extraordinary claims of novel physics made by certain UWB advocates [4]. By the early 1990's, however, terminology had converged towards the use of the term "ultra-wideband" or "UWB." The first patent with the exact phrase "UWB antenna" was filed on behalf of Hughes in 1993. Figure 1 shows the number of US Patent Applications filed in each year during 1993-2007 containing the exact search phrase "UWB antenna."

The 1990's were an era of only modest interest in UWB antennas, and only a few US patent applications were filed. In 1999, however, UWB became front-page news in USA Today and elsewhere [5]. By 2000, industry leaders like the Time Domain Corporation, Xtreme Spectrum, and others were engaging in active lobbying of the FCC to open up spectrum for UWB applications. Their efforts were rewarded by FCC's Preliminary Report and Order in 2002 [6].

A surge of UWB patents in 2000 accompanied this increased commercial interest. Interest waned somewhat in 2001, followed by yet another surge in 2002 when the FCC 


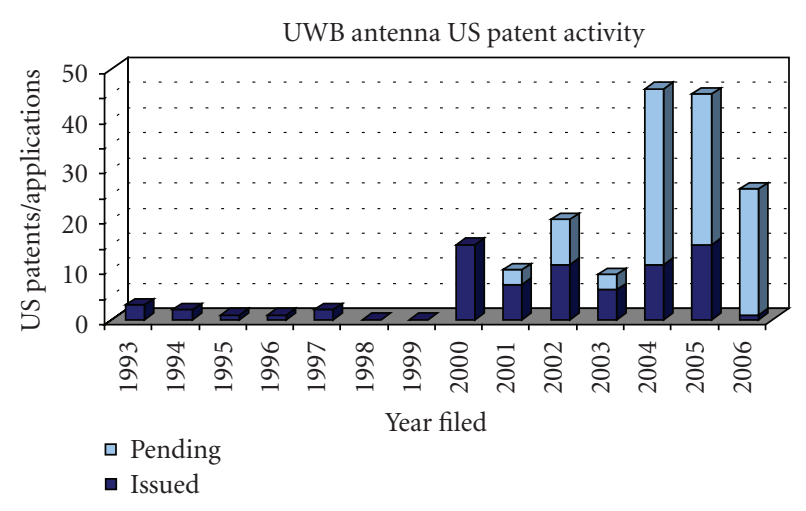

FIGURE 1: UWB antenna US patent activity by year (1993-2006).

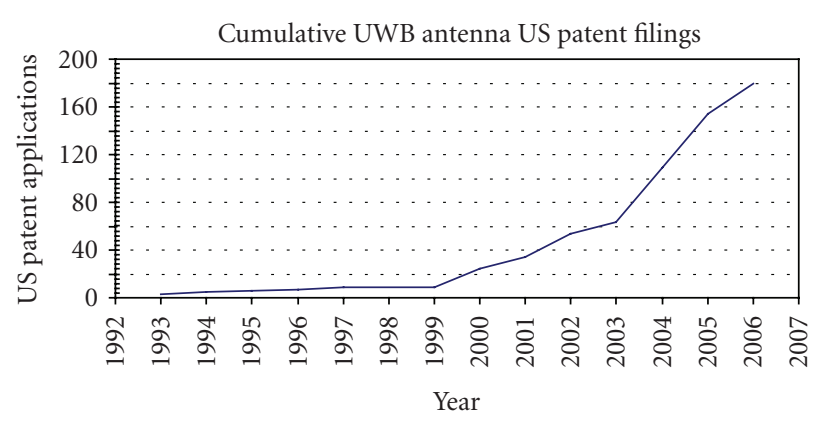

FIgURe 2: Cumulative UWB antenna US patent activity by year (1993-2006).

authorized UWB. Interest again waned in 2003 before exploding in 2004 and 2005. The pace of innovation dampened only slightly in 2006. Patents filed in 2007 may not yet have been published, so complete data is only available through 2006. The height of the bars in Figure 1 shows the total number of applications filed, some of which have been issued (dark portion) and some of which are still pending or were abandoned after publication (light portion). Clearly the US Patent Office is still dealing with a substantial backlog of UWB-related patents filed in 2004-2005. Figure 2 shows a cumulative plot of UWB antenna US patent filings.

UWB systems have opened up new dimensions of antenna design. Antennas have become an organic part of RF systems, providing filtering and other custom designed frequency-dependent properties. The wide bandwidths of UWB antennas present new challenges for design, simulation, and modeling. Optimizing UWB antennas to meet the demands of UWB propagation channels is similarly challenging. And as always, consumer applications demand compact and aesthetically pleasing designs that must nevertheless perform. Designers are meeting these challenges by extending the bandwidth of familiar antenna architectures like patches and slots. Designers are also using applying familiar techniques like arrays to UWB applications, as well as employing more recent concepts like antenna spectral filtering. The time is ripe for a special issue on UWB antennas that captures this progress and provides insight to where UWB antenna design will go in the future.

James Becker

Dejan Filipovic

Hans Schantz

Seong-Youp Suh

\section{REFERENCES}

[1] H. Schantz, The Art and Science of Ultrawideband Antennas, Artech House, Boston, Mass, USA, 2005.

[2] H. Harmuth, Antennas and Waveguides for Nonsinusoidal Waves, Academic Press, New York, NY, USA, 1984.

[3] C. A. Fowler, J. Entzminger, and J. Corum, "Assessment of ultrawideband (UWB) technology," IEEE Aerospace and Electronic Systems Magazine, vol. 5, no. 11, pp. 45-49, 1990.

[4] C. A. Fowler, "The UWB (impulse) radar caper or 'punishment of the innocent," IEEE Aerospace and Electronic Systems Magazine, vol. 7, no. 12, pp. 3-5, 1992.

[5] K. Maney, "Radical new digital technology may revolutionize communications," USA Today, April 1999.

[6] U.S. 47 C.F.R. Part 15 Subpart F Section 15.503d UltraWideband Operation. 

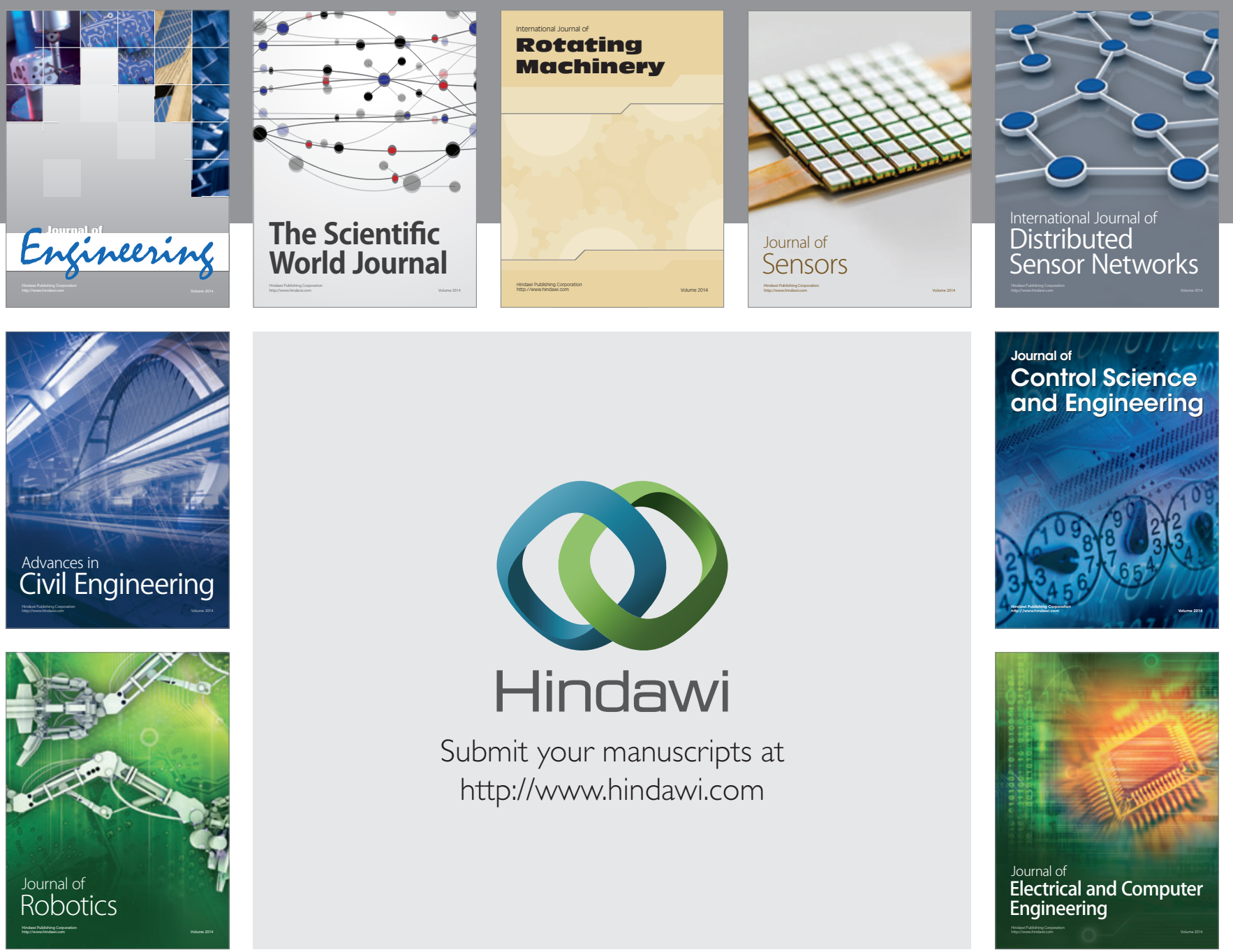

Submit your manuscripts at

http://www.hindawi.com
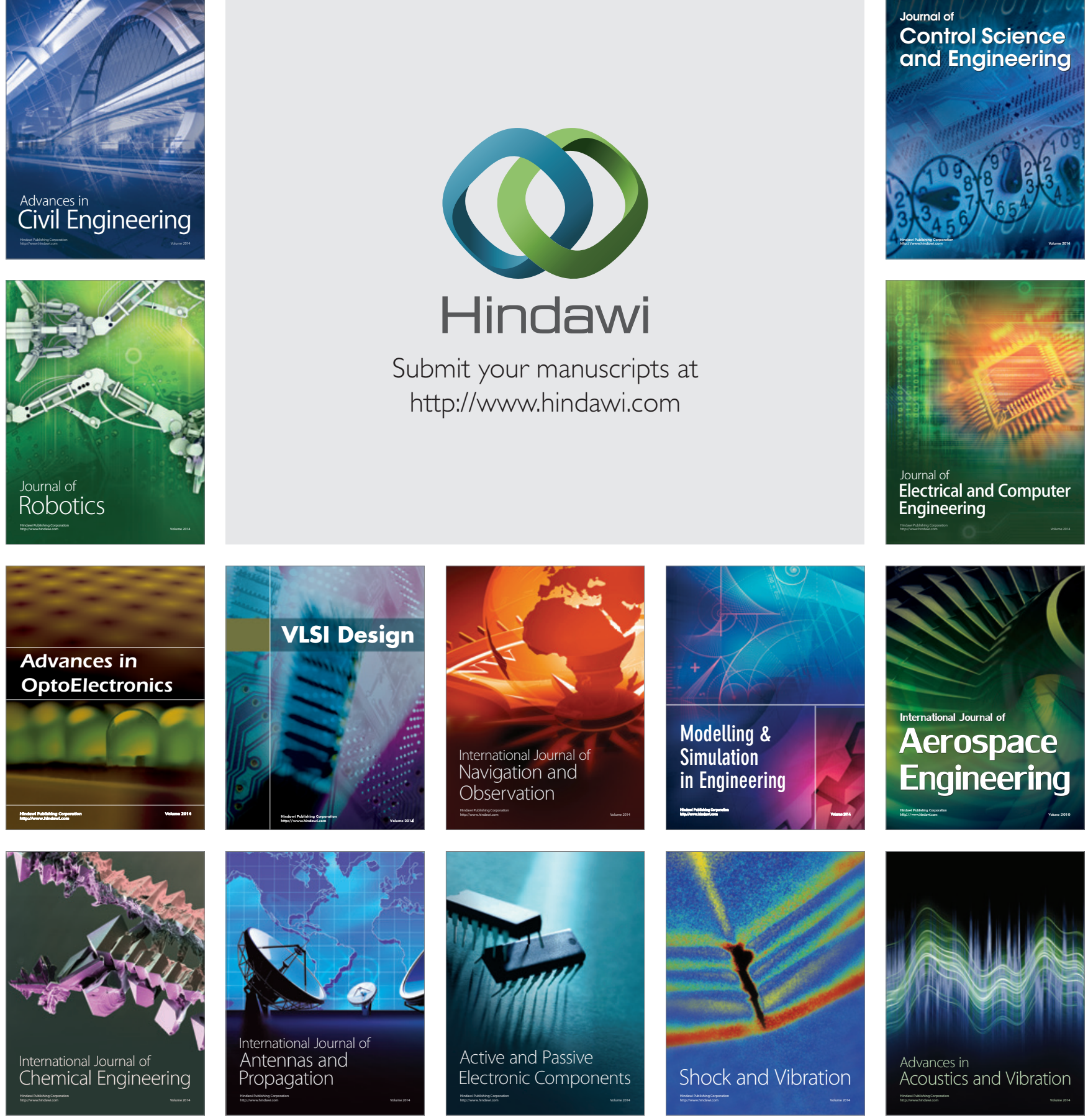